\title{
Effect of 5-Aza-2'-deoxycytidine on SLC22A18 in glioma U251 cells
}

\author{
SHENG-HUA CHU, YAN-BIN MA, DONG-FU FENG, HONG ZHANG, JIAN-HUA QIU and ZHI-AN ZHU \\ Department of Neurosurgery, No. 3 People's Hospital Affiliated with Shanghai Jiao Tong University \\ School of Medicine, Shanghai 201900, P.R. China
}

Received June 7, 2011; Accepted October 7, 2011

DOI: $10.3892 / \mathrm{mmr} .2011 .620$

\begin{abstract}
SLC22A18 [solute carrier family 22 (organic cation transporter) member 18] is located within the $11 \mathrm{p} 15.5$ cluster, and may be a new tumor suppressor gene; evidence of SLC22A18 hypermethylation is documented in several types of human cancers. In order to determine whether SLC22A18 hypermethylation is involved in glioma, we determined the SLC22A18 gene protein expression, mRNA expression and methylation status in glioma U251 cells before and after treatment with 5-Aza-2'-deoxycytidine (5-Aza-CdR), and observed the change in growth. Glioma U251 cells treated with 5-AzaCdR were analyzed by flow cytometry to identify any change in their cell cycle profiles. Tumors induced via the injection of untreated U251 cells were measured. Immunohistochemistry, reverse transcriptase-polymerase chain reaction (RT-PCR) and PCR-based methylation assay were carried out to determine SLC22A18 gene protein expression, mRNA expression and methylation status in glioma U251 cells before and after treatment with 5-Aza-CdR. The treated cells showed an increase in their proportion in G1, from 79.2 to $83.5 \%$, and a decrease in $\mathrm{S}$ phase, from 12.4 to $5.8 \%$. The apoptotic rate increased from 6.4 to $15.8 \%$. Tumors induced via the injection of untreated U251 cells were approximately $1.46 \mathrm{~cm}^{3}$ in size, whereas the tumors induced by $\mathrm{U} 251$ cells treated with 5 -Aza-CdR averaged $0.88 \mathrm{~cm}^{3}$ in size. The expression levels of SLC22A18 protein and mRNA in U251 cells were increased following treatment with $5 \times 10^{-7}$ M 5-Aza-CdR. Prior to 5-Aza-CdR treatment, the SLC22A18 gene demonstrated hypermethylation and therefore could not be cleaved by HpaII and MspI. It is known that only the DNA digested with HpaII or MspI can be amplified. Following treatment with 5-Aza-CdR, the SLC22A18 gene became demethylated, and could then be cleaved by both of the enzymes, and this failed to be amplified.5-Aza-cdR may induce glioma U251 cell division and apoptosis and enhance demeth-
\end{abstract}

Correspondence to: Dr Sheng-Hua Chu, Department of Neurosurgery, No. 3 People's Hospital Affiliated with Shanghai Jiao Tong University School of Medicine, Shanghai 201900, P.R. China E-mail: shenghuachu@126.com

Key words: solute carrier family 22 (organic cation transporter) member 18, 5-Aza-2'-deoxycytidine, demethylation, glioma ylation and protein and mRNA expression of SLC22A18. The hypermethylation of SLC22A18 may be related to the transcriptional silencing of this gene. The growth inhibitory effects of 5-Aza-CdR treatment in vivo remain recognizable.

\section{Introduction}

Gliomas are a major class of human intrinsic brain tumors. They include generally well-differentiated low-grade astrocytomas, anaplastic astrocytomas, and the most malignant brain tumor of adulthood, glioblastoma multiforme. Although the molecular mechanisms of glioma remain poorly understood, an increasing number of genetic abnormalities have been recognized; for example, the SLC22A18 [solute carrier family 22 (organic cation transporter) member 18] and the c-Met genes (1-3). We previously found that inactivation of the tumor-suppressor gene SLC22A18 was observed in a significant proportion of patients with glioma (1). An essential mechanism of gene inactivation is aberrant methylation in the promoter region of the gene (1). Evidence of SLC22A18 hypermethylation has also been documented in several types of human cancers (4-7). However, to our knowledge, there are few studies on the SLC22A18 gene in glioma. 5-Aza-2'deoxycytidine (5-Aza-CdR) is thought to act by inhibiting DNA methyltransferase that methylates cytosine residues in eukaryotic DNA (8). Several researchers have used it as an experimental tool for demethylation $(9,10)$. This drug appears to be incorporated into the cellular DNA where it acts as a non-competitive inhibitor of the maintenance methylase $(9,11)$. Methyl moieties are thus passively removed when the DNA undergoes replication and, indeed, numerous inactive cellular and viral genes are activated following exposure to 5-Aza-CdR (12). In order to determine whether SLC22A18 hypermethylation is involved in glioma, we determined the SLC22A18 gene protein expression, mRNA expression and methylation status in glioma U251 cells before and after treatment with 5-Aza-CdR, and observed the change in growth.

\section{Materials and methods}

Cell culture. U251 cells (human glioma cells from Wuhan University, China) were incubated at $37^{\circ} \mathrm{C}$ in RPMI-1640 medium supplemented with $10 \%$ calf serum, $100 \mathrm{U} / \mathrm{ml}$ penicillin and $100 \mu \mathrm{g} / \mathrm{ml}$ streptomycin, in an atmosphere of $5 \%$ 
$\mathrm{CO}_{2}$ at saturation humidity. The cell line was subcultured 2-3 days later, at an initial concentration of $5 \times 10^{4}$ cells $/ \mathrm{ml}$. Cells in logarithmic growth were used in all experiments.

5-Aza-CdR treatment. Cells $\left(\sim 1 \times 10^{5} / \mathrm{ml}\right)$ were plated in the vial and treated with $5 \times 10^{-7} \mathrm{M} 5$-Aza-CdR (Sigma Co.) for $24 \mathrm{~h}$. The medium was replaced following $24 \mathrm{~h}$ of drug treatment, and cells were cultured for 9 days, to ensure complete recovery from the immediate toxic effects of 5-Aza-CdR (13). Untreated cells were analyzed under similar conditions, as a control.

Determination of cell cycle profile. Cells $\left(2 \times 10^{6} / 100 \mathrm{ml}\right.$ vial $)$ were plated and treated with $5 \times 10^{-7} \mathrm{M} 5$-Aza-CdR. Cells were fixed after 7 days with $700 \mathrm{ml} / 1$ ethanol and stained with propidium iodide $(50 \mathrm{mg} / \mathrm{l}$; Crze Co.). DNA content at each cell cycle stage was determined via flow cytometry.

Effects of 5-Aza-CdR on tumorigenicity. U251 cells $\left(2 \times 10^{6}\right)$ were injected into the right flank of 4-week-old Balb/c male nude mice 10 days after treatment with 5-Aza-cdR. Untreated cells were injected into the left flank under the same conditions, as a control. Tumor sizes were measured 4 weeks after injection.

Immunohistochemistry. Cells were plated in 60-mm tissue culture dishes containing cover glasses overnight. The cover glasses were washed in PBS, fixed for $30 \mathrm{~min}$ in $70 \mathrm{ml} / 1$ ethanol and washed twice in PBS. Quenching of the endogenous peroxidase activity was obtained by treatment with $0.3 \mathrm{ml} / 1$ $\mathrm{H}_{2} \mathrm{O}_{2}$ in methanol. The sections were blocked with $10 \mathrm{ml} / \mathrm{l}$ goat serum in PBS and incubated with $3 \mu \mathrm{g}$ anti-SLC22A18 polyclonal antibody (Santa Cruz Biotechnology Inc., Santa Cruz, CA, USA) at $48^{\circ} \mathrm{C}$ overnight, then incubated with horseradish peroxidase (HRP)-conjugated antibody for $30 \mathrm{~min}$ at room temperature. Following three washes of $5 \mathrm{~min}$ in $\mathrm{PBS}$, they were developed in a substrate solution of HRP. A negative control test omitting the SLC22A18 antibody was also performed to confirm the absence of non-specific reactions. The manufacturer provides the presence (+) and absence (-) controls. The expression of the SLC22A18 gene was observed under a light microscope and analyzed using the figure analysis method.

Reverse transcriptase-polymerase chain reaction (RT-PCR). Total RNA was extracted from U251 cells by a modification of the guanidinium thiocyanate-acid phenol method and quantified based on the measured absorbance at $260 \mathrm{~nm}$. Complementary DNA (cDNA) was synthesized using $2 \mu \mathrm{g}$ RNA, $106 \mathrm{U} / 1$ reverse transcriptase (Gibco, Inc.) and $0.5 \mathrm{~g} / \mathrm{l}$ oligodeoxythymidine, in a total volume of $20 \mu \mathrm{l}$. The reaction was performed at $42^{\circ} \mathrm{C}$ for $60 \mathrm{~min}$ and terminated by heating at $99^{\circ} \mathrm{C}$ for $5 \mathrm{~min}$. Primer sequences and conditions for the RT-PCR product were previously described (forward primer, 5'-GCTTCGGCGTCGGAGTCAT-3' and reverse primer, 5'-AGCCTGGGCGTCAGTTTT-3') (14). The housekeeping gene GAPDH was used as an internal control for the $\mathrm{RT}$ reaction (forward primer, 5'-GGGAGCCAAAAGGGTCATCATCTC-3' and reverse primer, 5'-CCATGCCAGTGAGCTTCCCGTTC-3') (14). For amplification of cDNA, $2 \mu \mathrm{l}$ cDNA product was subjected to a PCR-based technique using 2.5 units of Taq DNA polymerase, $1 \mu \mathrm{l}$ each of forward and reverse primers and $200 \mathrm{lmol} / \mathrm{l}$ of each dNTP. PCR consisted of 35 cycles at $94^{\circ} \mathrm{C}$ for $1 \mathrm{~min}$, at $62^{\circ} \mathrm{C}$ for $1 \mathrm{~min}$ and at $72^{\circ} \mathrm{C}$ for $1 \mathrm{~min}$, followed by a final extension at $72^{\circ} \mathrm{C}$ for $5 \mathrm{~min}$. RT-PCR products were resolved on $2 \%$ agarose gel by the figure analysis method.

PCR-based methylation assay. DNA was isolated with the TriPure isolation reagent. A PCR assay relying on the inability of certain restriction enzymes, such as HpaII or MspI, to cut methylated sequences was used to analyze the methylation status of SLC22A18. DNA digests were performed according to the manufacturer's instructions (Boehringer Mannheim Co.). DNA (1 $\mu \mathrm{g})$ was digested for $2 \mathrm{~h}$ with 10 units of enzyme per $\mu \mathrm{g}$ DNA. Digested DNA (200 ng) was amplified with primers flanking the restriction sites. The primer set used for methylation analysis of SLC22A18 was 5'-CGTTTTTGTAAAGGTAGGTATTCGA-3' (sense) and 5'-AAACTAAAAAAAACAAAACAACCG-3' (antisense) (14). The amplification product was $146 \mathrm{bp}$. Conditions were: $94^{\circ} \mathrm{C}$ for $3 \mathrm{~min}, 35$ cycles at $94^{\circ} \mathrm{C}$ for $1 \mathrm{~min}, 57^{\circ} \mathrm{C}$ for $30 \mathrm{sec}$ and $72^{\circ} \mathrm{C}$ for $40 \mathrm{sec}$, followed by incubation at $72^{\circ} \mathrm{C}$ for $1 \mathrm{~min}$. PCR products were resolved on $2 \%$ agarose gel. To rule out the possibility of incomplete restriction, all samples were digested twice with each of the enzymes in independent experiments. PCR ampifications from each of the duplicate digests were repeated at least twice to ensure reproducibility of the results.

Statistical analysis. The data are expressed as the means \pm SE. The results were analyzed by the analysis of variance test. $\mathrm{P}<0.05$ was considered to denote statistical significance.

\section{Results}

Cell cycle arrest following treatment with 5-Aza-CdR. U251 cells treated with 5-Aza-CdR were analyzed by flow cytometry to identify any change in their cell cycle profiles. The treated cells revealed an increase in their proportion in G1, from 79.2 to $83.5 \%$, and a decrease in S phase, from 12.4 to $5.8 \%$. The apoptotic rate increased from 6.4 to $15.8 \%$. These results indicated that 5-Aza-cdR may induce cancer cell division and apoptosis.

Effects of 5-Aza-CdR on tumorigenicity. Tumors induced via the injection of untreated U251 cells were $\sim 1.46 \mathrm{~cm}^{3}$ in size, whereas the tumors induced by U251 cells treated with 5-Aza-CdR averaged $0.88 \mathrm{~cm}^{3}$ in size. This demonstrated that the growth-inhibitory effects of 5-Aza-CdR treatment in vivo were still recognizable.

Effect of 5-Aza-CdR on SLC22A18 protein expression. The positive 5-Aza-CdR-treated cells revealed mostly cytoplasmic, homogeneous and brown staining. Following treatment with $5 \times 10^{-7}$ M 5-Aza-CdR, U251 cells revealed a higher expression of SLC22A18 protein than untreated cells. We observed under a light microscope five fields in every slide at random, and measured the staining degree of every 124 positive cells by the figure analysis method. The IOD was $92.23 \pm 28.24$ and $382.74 \pm 42.35$, respectively, in the untreated and the 
A

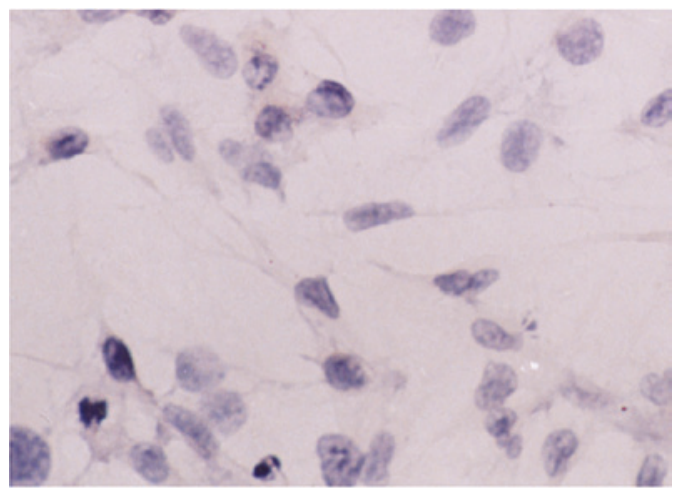

B

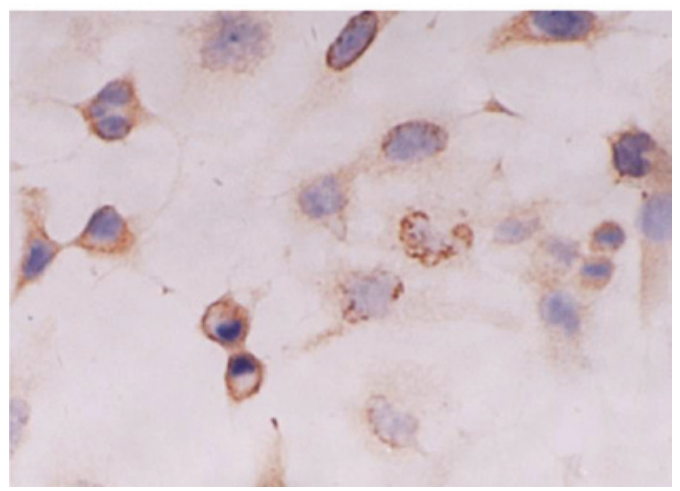

Figure 1. Immunohistochemical demonstration of SLC22A18 protein expression in U251 cells (SABC, x400). (A) Before treatment with 5-Aza-CdR; (B) after treatment with 5-Aza-CdR.

treated group. This revealed a significant difference $(\mathrm{P}<0.05)$. Although SLC22A18 protein expression alterations are complicated and still undefined, our data revealed that SLC22A18 protein expression could be silenced by hypermethylation of the SLC22A18 gene (Fig. 1).

Effect of 5-Aza-CdR on SLC22A18 mRNA expression. Fig. 2 reveals that the expression level of SLC22A18 mRNA in U251 cells was increased following treatment with $5 \times 10^{-7} \mathrm{M}$ 5-Aza-CdR. These results demonstrated a strong correlation between hypermethylation of SLC22A18 and the transcriptional silencing of this gene.

Effect of 5-Aza-CdR on SLC22A18 gene methylation status. Prior to 5-Aza-CdR treatment, the SLC22A18 gene revealed hypermethylation, and therefore could not be cleaved by HpaII and MspI. It is known that only DNA digested with $\mathrm{HpaII}$ or $M s p I$ can be amplified. Following treatment with 5-Aza-CdR, the SLC22A18 gene became demethylated, and could then be cleaved by both of the enzymes, and this failed to be amplified. Our experiment demonstrated that hypermethylation in SLC22A18 and 5-Aza-CdR enhanced demethylation (Fig. 3).

\section{Discussion}

Glioma is a deadly human tumor. Even with improved diagnosis and comprehensive therapy, the prognosis of glioma remains dismal (15). Studies of the underlying molecular mechanisms involved in glioma formation and progression provide tremendous opportunities to identify key molecules which may serve as additional targets for drug design in the treatment of brain

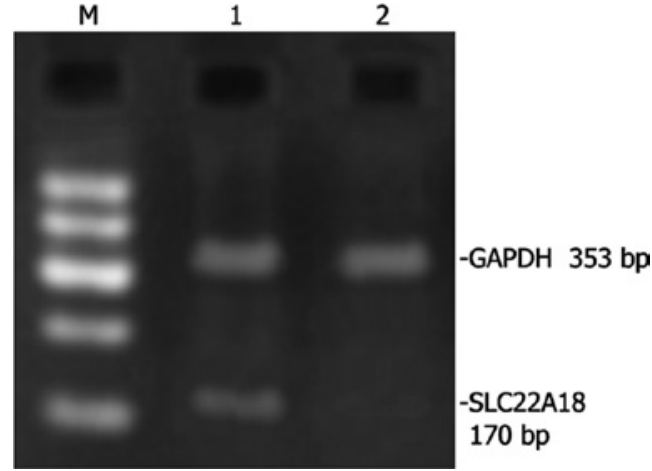

Figure 2. Effects of 5-Aza-cdR on SLC22A18 mRNA expression in U251 cells. Cells were treated with $5 \times 10^{-7}$ M 5-Aza-CdR for $24 \mathrm{~h}$; after culturing for 9 days, SLC22A18 mRNA was determined by RT-PCR. Lane M, the marker of standard DNA molecular mass; lane 1, treated with 5-Aza-CdR; lane 2, untreated.

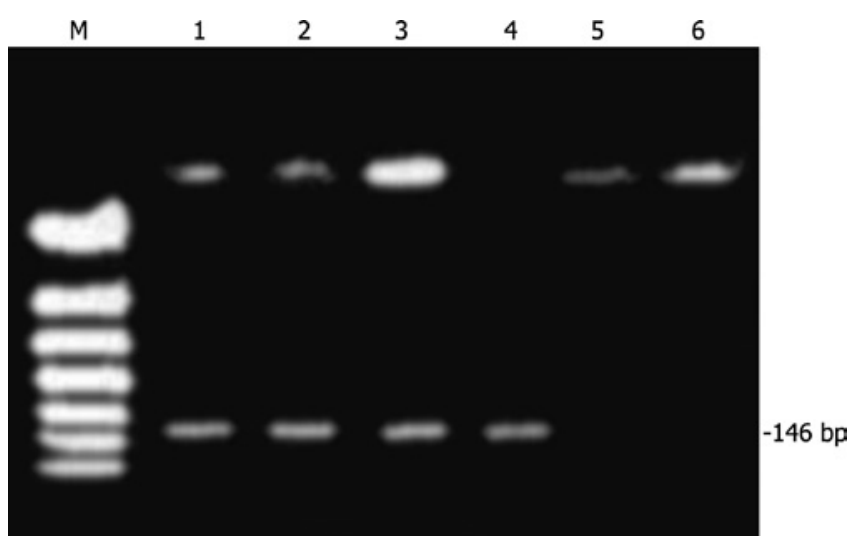

Figure 3. Detection of aberrant methylation of the SLC22A18 gene in glioma U251 cells. Lane M, molecular weight marker; lane 1, neither treated with 5-Aza-CdR nor digested with HpaII or MspI; lane 2, untreated with 5-Aza-CdR but digested with HpaII; lane 3, untreated with 5-Aza-CdR but digested with MspI; lane 4, treated with 5-Aza-CdR but not digested with HpaII or MspI; lane 5, treated with 5-Aza-CdR and digested with HpaII; lane 6, treated with 5-Aza-CdR and digested with MspI.

tumors. Mutations and overexpression of several oncogenes, including c-Met, PDGF and c-myc, have been identified in glioma patients $(3,16)$. Loss of heterozygosity has been found in several chromosomes in patients with glioma using molecular analysis. Many of these chromosomal segments contain known tumor-suppressor genes $(17,18)$, such as p16 on $9 p, p 53$ on $17 p$ and RB on 13q. More recently, SLC22A18 was located within the $11 \mathrm{p} 15.5$ cluster, and has been assigned a number of different nomenclatures $(4,5)$. Blast homology analysis suggests that SLC22A18 is a member of a family of polyspecific transporters and multidrug resistance genes (5). SLC22A18 has been shown to be a tumor-suppressor candidate and a substrate for RING105 (6). Structural mutations in SLC22A18 are rare, with isolated reports of point mutations in a breast cancer cell line, a rhabdomyosarcoma cell line (5), and Wilms' and lung tumors (4). Exonic deletions in Wilms' tumors and loss of heterozygosity in hepatoblastomas have also been reported $(4,7)$, indicating that SLC22A18 may play a role in tumorigenesis. We previously found that inactivation of tumor-suppressor gene SLC22A18 was described in a significant proportion of patients with glioma (1). An essential 
mechanism of gene inactivation is aberrant methylation in the promoter region of the gene (1). Evidence of SLC22A18 hypermethylation has also been documented in several types of human cancers (4-7). However, to our knowledge, there are few studies on the SLC22A18 gene in glioma.

Numerous investigations described reactivation of genes by 5 -Aza-CdR by acting on various loci of the inactive $\mathrm{X}$ chromosome (19) and the E-cadherin gene (20). In order to detect similar methylation changes in glioma U251 cells, we also used 5-Aza-CdR. In this study, U251 cells treated with 5-Aza-CdR revealed demethylation of the SLC22A18 gene. The SLC22A18 mRNA and protein levels were increased dramatically, the cell cycle was arrested in G1, the apoptotic rate increased and the implanted tumors grew more slowly. Our results show that: i) SLC22A18 hypermethylation is associated with glioma U251 cells; ii) MTase is increased in U251 cells; iii) the $5^{\prime} \mathrm{CpG}$ island of the SLC22A18 promoter was not methylated completely in glioma U251 cells; iv) the SLC22A18 gene may be reactivated by inhibiting the activities of MTase; v) SLC22A18 methylation may be a common event during the establishment of the cell line in vitro; and vi) the SLC22A18 gene encodes a cell cycle regulatory protein that belongs to the cyclin-dependent kinase inhibitory protein family and regulates the G1/S phase cell cycle transition; therefore, reactivated SLC22A18 may affect the growth of glioma U251 cells. The induction of SLC22A18 activation by 5-Aza-CdR in vivo and the decreased tumorigenicity observed in animal experiments suggest the chemotherapeutic potential of 5-Aza-CdR in the management of cancer.

\section{Acknowledgements}

This study was supported by a grant from the Natural Science Foundation of China (30901535), a grant from the Research Fund of Xinhua Hospital Affiliated with Shanghai Jiao Tong University School of Medicine (10XHJT01) and a grant from the New One Hundred Person Project of Shanghai Jiao Tong University of School of Medicine (10XBR01).

\section{References}

1. Chu SH, Ma YB, Feng DF, Qiu JH, Zhang H and Zhu ZA SLC22A18 gene expression and growth suppression of glioma cells. Chin J Exp Surg 28: 312, 2011.

2. Chu SH, Feng DF, Zhang H, Chen ET, Duan ZX, Li XY, Li J, Ma YB, Zhu ZA and Qiu JH: c-Met-targeted RNA interference inhibits growth and metastasis of glioma U251 cells in vitro. J Neurooncol 93: 183-189, 2009.

3. Chu SH, Ma YB, Feng DF, Zhang H, Qiu JH and Zhu ZA: c-Met antisense oligodeoxynucleotides increase sensitivity of human glioma cells to paclitaxel. Oncol Rep 24: 189-194, 2010.
4. Lee MP, Reeves C, Schmitt A, Su K, Connors TD, Hu RJ, Brandenburg S, Lee MJ, Miller G and Feinberg AP: Somatic mutation of TSSC5, a novel imprinted gene from human chromosome 11p15.5. Cancer Res 58: 4155-4159, 1998.

5. Schwienbacher C, Sabbioni S, Campi M, Veronese A, Bernardi G, Menegatti A, Hatada I, Mukai T, Ohashi H, Barbanti-Brodano G, Croce CM and Negrini M: Transcriptional map of $170-\mathrm{kb}$ region at chromosome 11p15.5: identification and mutational analysis of the BWR1A gene reveals the presence of mutations in tumor samples. Proc Natl Acad Sci USA 95: 3873-3878, 1998.

6. Yamada HY and Gorbsky GJ: Tumor suppressor candidate TSSC5 is regulated by UbcH6 and a novel ubiquitin ligase RING105. Oncogene 25: 1330-1339, 2006.

7. Albrecht S, Hartmann W, Houshdaran F, Koch A, Gärtner B, Prawitt D, Zabel BU, Russo P, von Schweinitz D and Pietsch T: Allelic loss but absence of mutations in the polyspecific transporter gene BWR1A on 11p15.5 in hepatoblastoma. Int J Cancer 111: 627-632, 2004.

8. Boivin AJ, Momparler LF, Hurtubise A and Momparler RL: Antineoplastic action of 5-aza-2'-deoxycytidine and phenylbutyrate on human lung carcinoma cells. Anticancer Drugs 13: 869-874, 2002

9. Yan WH, Lin AF, Chang CC and Ferrone S: Induction of HLA-G expression in a melanoma cell line OCM-1A following the treatment with 5-aza-2'-deoxycytidine. Cell Res 15: 523-531, 2005.

10. Liu LH, Xiao WH and Liu WW: Effect of 5-Aza-2'-deoxycytidine on the P16 tumor suppressor gene in hepatocellular carcinoma cell line HepG2. World J Gastroenterol 7: 131-135, 2001.

11. Nishioka C, Ikezoe T, Yang J, Komatsu N, Koeffler HP and Yokoyama A: Blockade of MEK signaling potentiates 5-Aza-2'deoxycytidine-induced apoptosis and upregulation of p21(waf1) in acute myelogenous leukemia cells. Int J Cancer 125: 1168-1176, 2009.

12. Patties I, Jahns J, Hildebrandt G, Kortmann RD and Glasow A: Additive effects of 5-aza-2'-deoxycytidine and irradiation on clonogenic survival of human medulloblastoma cell lines. Strahlenther Onkol 185: 331-338, 2009.

13. Bender CM, Pao MM and Jones PA: Inhibition of DNA methylation by 5-aza-2'-deoxycytidine suppresses the growth of human tumor cell lines. Cancer Res 58: 95-101, 1998.

14. Xu HM, Zhang HW, He HY, Hou YY and Zhao ZQ: Methylation and mRNA expression of imprinted gene SLC22A18/TSSC5/ BWR1A in breast cancer. Chin J Pathophysiol 24: 1007-1012, 2008

15. Kanu OO, Mehta A, Di C, Lin N, Bortoff K, Bigner DD, Yan H and Adamson DC: Glioblastoma multiforme: a review of therapeutic targets. Expert Opin Ther Targets 13: 701-718, 2009.

16. Dong Y, Jia L, Wang X, Tan X, Xu J, Deng Z, Jiang T, Rainov NG, Li B and Ren H: Selective inhibition of PDGFR by imatinib elicits the sustained activation of ERK and downstream receptor signaling in malignant glioma cells. Int J Oncol 38: 555-569, 2011.

17. Hu J, Jiang C, Ng HK, Pang JC, Tong CY and Chen S: Genomewide allelotype study of primary glioblastoma multiforme. Chin Med J 116: 577-583, 2003.

18. Squatrito M, Brennan CW, Helmy K, Huse JT, Petrini JH and Holland EC: Loss of ATM/Chk2/p53 pathway components accelerates tumor development and contributes to radiation resistance in gliomas. Cancer Cell 18: 619-629, 2010.

19. Jones PA, Taylor SM, Mohandas T and Shapiro LJ: Cell cyclespecific reactivation of an inactive $\mathrm{X}$-chromosome locus by 5-azadeoxycytidine. Proc Natl Acad Sci USA 79: 1215-1219, 1982

20. Yoshiura K, Kanai Y, Ochiai A, Shimoyama Y, Sugimura T and Hirohashi S: Silencing of the E-cadherin invasion-suppressor gene by $\mathrm{CpG}$ methylation in human carcinomas. Proc Natl Acad Sci USA 92: 7416-7419, 1995. 